\title{
Liderazgo directivo y cultura organizacional en la Universidad Peruana Los Andes
}

\author{
Leadership and organizational culture at the Universidad \\ Peruana Los Andes
}

\author{
Herbert Víctor Huaranga Rivera ${ }^{1,}$,, Miriam Angoma Astucuri ${ }^{2}$, Juan A. Picoy Gonzales ${ }^{3}$ \\ ${ }^{1}$ Universidad Nacional Daniel Alcides Carrión, ${ }^{2}$ Universidad Peruana Los Andes, ${ }^{3}$ Universidad Nacional Hermilio Valdizan
}

\section{RESUMEN}

El objetivo fue determinar la correlación que existe entre el liderazgo directivo y la cultura organizacional en los docentes de la Universidad Peruana Los Andes, sede La Merced - 2017. La investigación fue de tipo aplicada, nivel correlacional y se utilizó el método descriptivo, hipotético deductivo, con un diseño descriptivo - correlacional, la muestra fue probabilística de 113 docentes. Del procesamiento de datos se infiere que en la variable liderazgo directivo el $65,49 \%$ perciben que el liderazgo directivo es bueno; luego el $30,09 \%$ perciben el liderazgo como muy bueno y el $4,42 \%$ perciben el liderazgo como deficiente. Asimismo, en la variable cultura organizacional, el $60,18 \%$ perciben una cultura favorable, luego el $25,66 \%$ perciben una cultura muy favorable, el $14,16 \%$ perciben una cultura desfavorable y ningún docente percibe como una cultura organizacional muy desfavorable. El nivel de liderazgo directivo predominante en la percepción de los docentes, consideran el nivel bueno siendo este el $56,86 \%$, luego muy bueno el $38,64 \%$ y deficiente el $4,55 \%$. El nivel de cultura organizacional percibido por los docentes es favorable en el $55,68 \%$, luego muy favorable en el $28,41 \%$, y finalmente muy desfavorable en el $15,91 \%$. Se concluye, con un nivel de significancia del $5 \%$ que existe una relación directa fuerte ("rho" $=0,856)$ y altamente significativa ( $p$-valor: $0,000<0,010$ ) entre el liderazgo directivo y la cultura organizacional en los docentes de la Universidad Peruana Los Andes, sede La Merced-2017.

Palabras clave: Liderazgo directivo, cultura organizacional, comunicación y condiciones laborales.

\begin{abstract}
The objective was to determine the correlation that exists between executive leadership and organizational culture in teachers of Universidad Peruana Los Andes, headquarters La Merced - 2017. The research was of the type applied, correlation level and method was used descriptive, hypothetical deductive, descriptive design - correlational, the sample was probabilistic of 113 teachers. From data processing, it can be inferred that in the directive leadership variable $65,49 \%$ perceive that managerial leadership is good; then $30,09 \%$ perceive leadership as very good and $4,42 \%$ perceive leadership as deficient. Also, in the organizational culture variable $60,18 \%$ perceive a favorable culture, then $25,66 \%$ perceive a very favorable culture, $14,16 \%$ perceive an unfavorable culture and no teacher perceives a very unfavorable organizational culture. The level of predominantly managerial leadership in the perception of teachers, consider the good level being this $56,86 \%$, then very good $38,64 \%$ and deficient $4,55 \%$. The level of organizational culture perceived by teachers is favorable at $55,68 \%$, then very favorable at $28,41 \%$, and finally very unfavorable at $15,91 \%$. It is concluded, with a level of significance of $5 \%$ that there is a strong direct relationship ("rho" $=0,856$ ) and highly significant ( $p$-value: $0,000<0,010$ ) between the managerial leadership and the organizational culture in teachers of Universidad Peruana Los Andes, headquarters La Merced - 2017.
\end{abstract}

Keywords: Executive leadership, organizational culture, communication and working conditions.

Historial del artículo:

Recibido, 04 de marzo 2018; aceptado, 11 de mayo de 2018; disponible en línea, 05 de julio de 2018

* Docente de la Universidad Nacional Daniel Alcides Carríon, Dr. en Contabilidad y Dr. en Educación.

Correo: Huancayo_huaranga@hotmail.com 


\section{INTRODUCCIÓN}

La presente investigación se enfoca desde una perspectiva a nivel internacional, ya que los problemas sobre la cultura organizacional son comunes en las organizaciones educativas del nivel superior. Si bien es cierto la gestión de las organizaciones está presente en todas las entidades universitarias, la misma que facilita el cumplimiento de los objetivos educacionales, también es necesario contar con un buen liderazgo directivo y una cultura organizacional adecuada.

Asimismo, a nivel nacional -según la reforma del currículo por competencias que impulsa el Ministerio de Educación- dentro de sus políticas ha priorizado las condiciones del clima en el aula y en las entidades educativas, dando énfasis a la gestión centralizada y los aprendizajes en donde todo cuerpo directivo, jerárquico y docente de las organizaciones educativas laboren en sintonía a las buenas relaciones humanas. Estos cambios ocasionan desajustes especialmente en las actuales autoridades educativas de las universidades a nivel nacional, tal es el caso de la Universidad Peruana Los Andes.

A nivel local y/o institucional, se ha podido observar que la comunidad de la Universidad Peruana Los Andes, tienen dificultades de manera directa en sus relaciones humanas, ya que como se ha podido apreciar, en casi todas las entidades universitarias públicas y privadas de nuestro país existen grupos focalizados que luchan por el poder, comenzando desde el proceso de planificación, ejecución y evaluación de las actividades académicas y de gestión.

De otro lado la interacción de los agentes educativos, su forma de organización y administración que se da función a la Ley Universitaria 30220; el cual se manifiesta en su desorganización cuando estos toman sus decisiones al interior, asimismo el ambiente imperante viene a ser en muchos casos el autoritarismo principalmente en las universidades privadas, tal es el caso de la Universidad Peruana Los Andes, y el trato entre el personal directivo (Coordinador de la sede La Merced y su súper estructura) con los docentes en la mayoría de los casos es horizontal, pero tiende al verticalismo.

Todos estos aspectos también se encuentran detallados y documentados en el Plan Estratégico de la Universidad, recopilados lógicamente mediante el Análisis DAFO, la Técnica del Pert CPM y el diagrama causal de Forest.

Frente a ello el estudio de liderazgo directivo y la cultura organizacional es importante, ya que esta contribuye a superar dichas dificultades que afectan a la Universidad Privada. Por lo tanto, se formula las siguientes preguntas de investigación.
¿Qué relación existe entre el liderazgo directivo y la cultura organizacional en los docentes de la Universidad Peruana Los Andes, sede La Merced en el periodo 2017 ?

El objetivo fue determinar la correlación que existe entre el liderazgo directivo y la cultura organizacional en los docentes de la Universidad Peruana Los Andes, Sede La Merced en el periodo 2017.

La hipótesis general fue: Existe correlación directa y significativa entre el liderazgo directivo y la cultura organizacional en los docentes de la Universidad Peruana Los Andes, Sede La Merced en el periodo 2017.

Según Alvarez (2007) el vocablo "líder" probablemente sea uno de los más revisados por la literatura contemporánea. Basta con detenerse a echar una mirada a revistas, periódicos, libros y publicaciones para comprobar hasta qué punto se ha extendido y generalizado su uso. Esto ha provocado, mucha ambigüedad en el uso del término que se emplea indistintamente. Se logra entender lo mismo cuando calificamos como líder a un dirigente político, empresarial o religioso. Según Lazzati (2011) el liderazgo es la actividad de influenciar en la gente para que se empeñe voluntariamente en el logro de los objetivos del grupo. Por grupo debe entenderse un grupo pequeño, un sector de la organización, una organización o una nación.

Respecto al liderazgo directivo surge a partir de $M c$ Gregor Burns y Bernad Bass, que describieron una serie de cualidades que conformaban un tipo de liderazgo empresarial que se comenzaba a valorar más en el mundo de la empresa. Se trataba del concepto de liderazgo directivo. En si el proceso común de líder y trabajadores para avanzar a un nivel más alto de la moral y la motivación. Es una transformación que produce cambios significativos en la empresa y en las personas que la conforman.

Por lo tanto, el liderazgo directivo, es definido como el proceso de influir, guiar y dirigir a los miembros de un grupo u organización, quienes siguen al líder de forma voluntaria en la búsqueda del éxito en la consecución de unos objetivos. Se han realizado extensos estudios sobre la materia y los investigadores han diferenciado varios tipos de liderazgo, siendo el transformacional uno de los que más atención ha recibido.

El concepto de liderazgo directivo fue originado e introducido por el experto en liderazgo Bass (1997), éste lo definió como el tipo de liderazgo ostentado por aquellos individuos con una fuerte visión y personalidad, gracias a la cual son capaces de cambiar las expectativas, percepciones y motivaciones, así como liderar el cambio dentro de una organización. 
Asimismo, determinó que tal tipología de liderazgo era observable "cuando los líderes y seguidores trabajan juntos para avanzar a un nivel superior de moral y motivación" (p. 144).

Las dimensiones del Liderazgo directivo, a continuación, se desarrollan las dimensiones del Liderazgo directivo. Según Bass (1997) el líder directivo se distingue por tres dimensiones básicas, relacionándolas con la labor desempeñada por el docente: autocrático, democrático y permisivo.

Por otra parte, la cultura organizacional es el nombre dado por diversos autores, al ambiente generado por las emociones de los miembros de un grupo u organización, el cual está relacionado con la motivación de los empleados. Se refiere tanto a la parte física como emocional. El concepto se asimila al de dinámica de grupo al analizar las fuerzas internas que inciden en el ambiente laboral como resistencia al cambio. Fue el sociólogo Kurt Lewin citado por Morán, (2007) quien desarrolló un análisis del campo de fuerzas, como modelo con el cual describía cualquier nivel presente de rendimiento.

Muchos investigadores consideran la cultura organizacional como las percepciones colectivas e individuales que tienen los trabajadores sobre su organización, influenciadas por variables psicosociales, laborales y organizacionales, que repercuten sobre el comportamiento organizacional y la productividad empresarial.

De esta definición se puede afirmar que la cultura organizacional es un concepto que está referido a la vida organizacional en una empresa. Es así, que la cultura organizacional es configurada como una dimensión o ámbito evaluativo básico y es definido como: "La calidad del entorno global de una organización" (Barba, 2011, p. 66).

Para Alvarado (2003, p.45) la cultura organizacional comprende la suma de las percepciones que describe el grado de satisfacción de las personas, de su medio laboral en que se desarrolla el trabajo cotidiano. Corroborando lo mencionado se reafirma, que es una percepción que se tiene de la persona y del medio ambiente laboral y consiste en el grado favorable o desfavorable del entorno laboral para las personas que integran la organización. La existencia de algunas categorías o dimensiones existentes de la cultura organizacional en una organización se relacionan con propiedades propias de cada uno. Es necesario precisar que dichas categorías no son únicas, como tampoco existe un cultura único o ideal depende de las características propias de cada entidad. Según Rodríguez (1999), la comunicación, las condiciones laborales, el involucramiento laboral, la autorrealización y la supervisión.

\section{MATERIAL Y MÉTODOS}

La investigación por su naturaleza fue del tipo aplicada y de nivel correlacional.

En la investigación, se utilizó el método general científico y como métodos específicos, el método descriptivo y el hipotético deductivo (Oseda y Mendivel, 2017).

El diseño de investigación utilizado fue el no experimental, descriptivo correlacional. Según (Kerlinger y Lee, 2002) el diseño descriptivo correlacional, es aquel diseño donde no se manipula ninguna variable, solo se miden y luego se comparan para ver qué correlación existe entre éstas variables. El esquema es el siguiente:

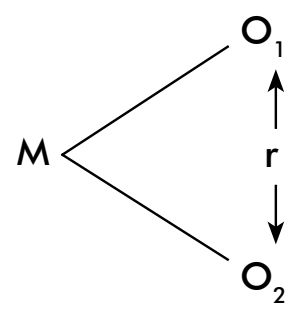

Donde:

$M=$ Muestra

$\mathrm{O}_{1}=$ Observación de la variable 1 .

$\mathrm{O}_{2}=$ Observación de la variable 2 .

$r=$ Correlación entre dichas variables.

En el caso de nuestra investigación, la población estuvo conformada por 140 docentes de la Universidad Peruana Los Andes, Sede La Merced-2017. El Muestreo fue probabilístico, y estuvo conformado por 113 docentes de la Universidad Peruana Los Andes.

Respecto a las técnicas de recolección de datos se tiene a la encuesta con su instrumento el cuestionario el cual se aplicó a las dos variables de estudio. La confiabilidad se hizo por el método de mitades partidas $(M M P=0,956)$ y la validez por el juicio de experto $(\%=96)$.

\section{RESULTADOS}

Según la tabla 1, se puede apreciar que los niveles de la variable 1 Liderazgo directivo de los 88 docentes encuestados, 74 docentes que representa el $(65,49 \%)$ perciben que el liderazgo directivo es bueno; luego 34 docentes que representan el $(30,09 \%)$ perciben el liderazgo directivo como muy bueno; luego 5 docentes que es el $(4,42 \%)$ perciben el liderazgo directivo como deficiente. En el nivel muy deficiente no se tuvo a 
Tabla 1

Niveles del Liderazgo directivo.

\begin{tabular}{lcr}
\hline \multicolumn{1}{c}{ Niveles } & Frecuencia & Porcentaje \\
\hline Muy deficiente & 0 & $0.00 \%$ \\
Deficiente & 5 & $4.42 \%$ \\
Bueno & 74 & $65.49 \%$ \\
Muy bueno & 34 & $30.09 \%$ \\
$\quad$ Total & 113 & $100.00 \%$ \\
\hline
\end{tabular}

Fuente: Bases de datos de los investigadores.

ningún docente de la Universidad. Como se puede inferir, el nivel predominante fue el nivel bueno.

Según la tabla 2, se puede apreciar que los niveles de la variable 2 "Cultura organizacional" de los 113 docentes encuestados, 68 docentes que representan el $(60,18 \%)$ perciben un cultura organizacional favorable; luego 29 docentes que representan el $(25,66 \%)$ perciben un cultura organizacional favorable; luego 16 docentes que son el $(14,16 \%)$ perciben un cultura organizacional desfavorable y ningún docente percibe

Tabla 2

Niveles de Cultura Organizacional.

\begin{tabular}{lcr}
\hline \multicolumn{1}{c}{ Niveles } & Frecuencia & Porcentaje \\
\hline Muy desfavorable & 0 & $0.00 \%$ \\
Desfavorable & 16 & $14.16 \%$ \\
Favorable & 68 & $60.18 \%$ \\
Muy favorable & 29 & $25.66 \%$ \\
$\quad$ Total & 113 & $100.00 \%$ \\
\hline
\end{tabular}

Fuente: Bases de datos de los investigadores.

que la cultura organizacional sea muy desfavorable. A manera de síntesis, el nivel predominante en esta variable fue el nivel favorable.

Respecto a la prueba de hipótesis, como el diseño utilizado fue el descriptivo - correlacional, el estadígrafo utilizado fue el coeficiente de correlación de Spearman, prueba bilateral $\circ$ a dos colas. Ahora bien, como la rho=0,854 y con un ( $p$-valor: $0,000<0,010)$ se rechaza la $\mathrm{Ho}$, y se acepta la hipótesis alterna $\mathrm{Hl}$; que dice: Se concluye que existe una correlación directa y altamente significativa entre el liderazgo directivo y la cultura organizacional en los docentes de la Universidad Peruana Los Andes, Sede La Merced- 2017.

\section{DISCUSIÓN}

Según la aplicación de los dos cuestionarios encuesta en base a la percepción docente sobre el liderazgo directivo y la cultura organizacional en los docentes de la Universidad Peruana Los Andes, sede La Merced2017.
Contrastando el problema o dificultad que presentan la mayoría de las universidades privadas en el país, según la muestra de estudio que es la Universidad Peruana Los Andes Sede La Merced en el periodo 2017 con los problemas o dificultades que se han investigado en otras realidades, para encontrar si es que existen semejanzas o diferencias, se encontró en relación con la variable $N^{\circ} 1$ Liderazgo directivo los resultados son los siguientes: un gran porcentaje percibe un nivel bueno $(65,49 \%)$, muy bueno $(30.09 \%)$ y deficiente el $(4,42 \%)$.

En consecuencia, de ello se afirma que el liderazgo directivo en la Universidad Peruana Los Andes es bueno y/o aceptable en la percepción de los docentes, pero se debe tener en cuenta la percepción de aquellos docentes que no la consideran favorable.

Estos resultados refuerzan lo encontrado en los antecedentes de la tesis de Ruiz (2011) cuando concluye que el estilo de liderazgo del director (Rector - Presidente de la Comisión de Gobierno) influye significativamente en la eficacia de las instituciones educativas del Consorcio "Santo Domingo de Guzmán" de Lima Norte.

En los datos obtenidos en la variable 2 Cultura organizacional se evidencia en mayor porcentaje en el nivel favorable siendo este el $60,18 \%$ y muy favorable $25,66 \%$ respectivamente en la percepción de los mismos docentes y en los niveles desfavorable 14,16\% y muy desfavorable $0.00 \%$.

Como consecuencia de ello se afirma que la cultura organizacional en la Universidad Peruana Los Andes es aceptable en la percepción de los docentes, pero se debe tener en cuenta la percepción de aquellos docentes que no la consideran favorable, aunque es un pequeño grupo, pero debe tenerse en cuenta.

Estos resultados son corroborados con los encontrados en los antecedentes en la tesis de Montalvo (2008) denominado: Relación entre cultura organizacional y el desempeño docente donde el $51 \%$ de los sujetos perciben la cultura organizacional en un alto nivel, muy similar a los encontrados en la presente investigación.

Respecto a la hipótesis general y estadística de investigación dice literalmente: Existe una relación directa y significativa entre el liderazgo directivo y la cultura organizacional en los docentes de la Universidad Peruana Los Andes, Sede La Merced2017. En el coeficiente de correlación entre la variable liderazgo directivo y la cultura organizacional, la "rho" de Spearman $=0,856$ con el nivel de significancia de $5 \%$ este es considerado como correlación positiva fuerte y en la decisión estadística se evidencia que el (p-valor: $0,000<0,010)$, en consecuencia, se rechaza la hipótesis nula (Ho) y se acepta la hipótesis alterna 
(Hi). Se concluye que existe una correlación directa fuerte y significativa entre el liderazgo directivo y la cultura organizacional en los docentes de la Universidad Peruana Los Andes, Sede La Merced2017.

Dichos resultados son corroborados en los encontrados en los antecedentes de la tesis de Montalvo (2008) cuando concluye que existe una relación entre el cultura organizacional y el nivel de desempeño docente, que es percibido por el docente y los estudiantes; el $51 \%$ de los sujetos encuestados percibe el cultura organizacional en un alto nivel, así como el $64 \%$ de los entrevistados percibe el desempeño docente en el nivel alto respectivamente y efectuar la correlación entre el liderazgo de los directores y la planificación de la enseñanza, se demuestra que existe una correlación moderada, la relación se expresa en un $65 \%$.

Asimismo, se ha visto que en las últimas décadas se ha mencionado mucho como el liderazgo ejerce influencia sobre sus subordinados. Esta es una condición humana básica y global, pues desde los inicios del ser humano donde ya vivían en sociedad han existido líderes que han guiado a los demás, de tal manera que a medida que la sociedad ha ido evolucionando, el liderazgo también se ha transformado (Barroso, 2010).

Podríamos asumir que el comportamiento del líder influye en sus seguidores, siendo el principal responsable del éxito o fracaso de ellos, que son los encargados de ayudar al cumplimiento de los objetivos organizacionales (Morán, 2007) expresado de otra manera el líder tiene en su poder el progreso de las organizaciones, ya que todas necesitan de alguien para ser dirigidas.

Asimismo, para Ponce (2014) la cultura organizacional influye directamente en el proceso y desarrollo de las funciones de las organizaciones, es decir la percepción que tiene el talento humano del lugar en el que se desempeña que influye en su actuar". Estas percepciones son las que le dan vida a la cultura organizacional y la gestión de la dirección cumple el papel más importante para le generación de un cultura desfavorable o favorable, por eso se dice que la existencia real de un clima está dada por la subjetividad de las personas que laboran en una organización.

Respecto al liderazgo directivo es uno de los factores más determinantes e influyentes en la percepción que tienen los trabajadores de la cultura organizacional (Ponce, 2014) y dada la importancia que tiene la cultura organizacional en el cumplimiento de los objetivos de la organización, una cultura positiva genera un mayor sentimiento de pertinencia hacia la organización provocando automáticamente un mejor desempeño en los trabajadores.
Para Peraza (2004) por el contrario, una cultura negativa disminuye el desempeño ocasionando bajo rendimiento y situaciones de conflicto. La presente investigación considera una revisión teórica del liderazgo, sus antecedentes, conceptos y teorías y a través del análisis de estudios previos determinar su relación sobre la cultura organizacional.

\section{Algunas conclusiones son:}

Se ha determinado con un nivel de significancia del $5 \%$ que existe una relación directa fuerte ("rho" $=0,856$ ) y altamente significativa ( $p$-valor: $0,000<0,010$ ) entre el liderazgo directivo y la cultura organizacional en los docentes de la Universidad Peruana Los Andes, sede La Merced- 2017; es decir a mayor liderazgo directivo, mejor será la cultura organizacional de la Universidad. El nivel de liderazgo directivo predominante en la percepción de los docentes de la Universidad Peruana Los Andes de sede La Merced- 2017, consideran el nivel bueno siendo este el $56,86 \%$, luego muy bueno el $38,64 \%$ y deficiente el $4,55 \%$.

El nivel de cultura organizacional percibido por los docentes de la Universidad Peruana Los Andes de sede La Merced - 2017, es favorable en el 55,68\%, luego muy favorable en el $28,41 \%$, y finalmente muy desfavorable en el 15,91\%.

\section{REFERENCIAS BIBLIOGRÁFICAS}

Alvarez, J. C., (2007). Administración y gestión estratégica. México: Interamericana.

Alvarado, J. J. (2003). Influencia del liderazgo en la competitividad de las organizaciones peruanas. Lima: PUCP.

Barba, D. (2011). El clima de trabajo, generado por la cultura organizacional y su influencia como determinante en el comportamiento del personal docente y administrativo de la Escuela Superior de Educación Física de la UAS. México: Universidad Auitónoma Metropolitana de México.

Barroso, F. (2010). Liderazgo y cultura organizacional en maquiladoras textiles de exportación. México: Interamericana.

Bass, B. (1997). Improving Organizacional Effectives Though Transformational. Leadership. New York: Sage.

Kerlinger, F. y Lee, H. (2002). Investigación del comportamiento. México: Interamericana.

Lazzati, M. (2011). Liderazgo de directivos contemporáneos. Lima: Ed. San Marcos.

Montalvo, L. (2008). El clima organizacional y su influencia en el desempeño docente en las instituciones educativas del nivel de educación secundaria de la UGEL 15 de Huarochirí-2008. 
Lima: Universidad Nacional de Educación.

Morán, A. (2007). Liderazgo en la función directiva. México: Mc Graw Hill.

Oseda, D. y Mendivel, R. K. (2017). Fundamentos de investigación científica. Lima: San Marcos.

Peraza, Y. (2004). Cultura organizacional: Conceptos y experiencias. Transporte Desarrollo y Medio Ambiente. México: Interamericana.

Ponce, P. (2014). Liderazgo femenino y cultura organizacional, en un instituto universitario. México: Mc Graw Hill.

Rodríguez, D. (1999). Liderazgo Gerencial. Bogotá: Mc Graw Hill.

Ruiz, P. (2011). Influencia del estilo de liderazgo del director en la eficacia de las instituciones educativas del consorcio Santo Domingo de Guzmán de Lima Norte. Lima: Universidad Nacional Mayor de San Marcos. 